\title{
Nonlocal multiscale modeling of deformation behavior of polycrystalline copper by second-order homogenization method
}

\section{Makoto Uchida \& Yoshihisa Kaneko}

\begin{tabular}{|c|c|}
\hline Citation & The European Physical Journal B. 92(9); 189 \\
\hline Issue Date & 2019-09-02 \\
\hline Type & Journal Article \\
\hline Textversion & author \\
\hline Relation & $\begin{array}{l}\text { This is a post-peer-review, pre-copyedit version of an article published in The } \\
\text { European Physical Journal B. The final authenticated version is available online } \\
\text { at: https://doi.org/10.1140/epjb/e2019-100231-4. }\end{array}$ \\
\hline DOI & 10.1140/epjb/e2019-100231-4 \\
\hline
\end{tabular}

\section{Self-Archiving by Author(s)}

Placed on: Osaka City University Repository 


\title{
Nonlocal Multiscale Modeling of Deformation Behavior of Polycrystalline Copper by Second-Order Homogenization Method
}

\author{
Makoto Uchida $^{1^{*}}$ and Yoshihisa Kaneko ${ }^{1}$ \\ 1 Graduate School of Engineering, Osaka City University, Osaka, Japan \\ * Graduate School of Engineering, Osaka City University, Sugimoto 3-3-138, Sumiyoshi-ku, Osaka, 558-8585, Japan \\ Tel: +81-6-6605-2673, Email: uchida@osaka-cu.ac.jp
}

\begin{abstract}
:
Engineering materials usually exhibit heterogeneity such as that observed in the polycrystalline structure of metals, and this heterogeneity affects the nonuniform deformation of a material. In this study, the micro- to macroscopic nonuniform deformation of polycrystalline copper specimen with a curved gage section is evaluated by a finite element method (FEM) simulation based on the second-order homogenization method (2nd-HM). The effects of the microstructure size and macroscopic stress gradient on the nonuniform deformation of the material are then investigated by comparing the simulation and experimental results. A two-dimensional plane strain polycrystalline microstructure was periodically applied to all the integration points in the macrostructure; the anisotropic deformation of the crystal grains is represented by the conventional crystalline plasticity constitutive equation. The computational results indicate that the interaction between nonuniform deformation on the micro and macroscopic scales induces a slight size effect in the material. However, the FEM simulation based on the 2nd-HM could not predict the decrease in the macroscopic strain concentration in the specimens with large crystalline grains, which was observed in the experimental studies, because of random strain localization resulting from the microscopic heterogeneity.
\end{abstract}

Keywords: polycrystalline copper, nonuniform deformation, homogenization method, size effect

\section{INTRODUCTION}

It is very important to evaluate and model the nonuniform deformation of engineering materials in the continuum mechanics of solids. Engineering materials usually exhibit heterogeneity such as that observed in the polycrystalline structure of metals, and this heterogeneity affects the nonuniform deformation of a material. The nonuniform deformation of various materials can be precisely evaluated by optical measurement methods, e.g., digital image correlation (DIC). The main concept of DIC was proposed by Sutton [1, 2], and many related papers have been published, e.g., [3-5]. The author's research group has also quantified the micro and macroscopic nonuniform deformations of polycrystalline copper using DIC [6-8]. A method for evaluating the strain and stress fields using the virtual field method has also been established $[9,10]$.

Quantitative information about the strain and stress fields facilitates the development of a theoretical model of 
the nonlocal mechanical behavior of engineering materials. To directly use the experimentally obtained strain and stress fields to model nonuniform deformation, physical quantities characterizing these fields should be included in the constitutive equation of the material. The strain gradient is usually employed as a barometer of nonuniform deformation. Furthermore, its work-conjugate higher-order stress is sometimes introduced to predict the nonuniform deformation of materials.

Constitutive models including the strain gradient (and its work-conjugate higher-order stress) have been developed in nonlocal continuum mechanics. The development of a framework for nonlocal continuum mechanics began in the 19th century. The Cosserat brothers [11] described nonlocal elastic deformation behavior involving a rotation gradient by introducing the couple stress. In the 1960s, Toupin [12] and Mindlin [13, 14] developed more general gradient theories of elastic deformation behavior. Since the 1980s, the strain gradient theory has been extended to plastic deformation, and various engineering problems have been studied using the gradient theory.

One of the major streams of strain gradient plasticity was developed to describe the shear band and deformation localization of materials showing softening during plastic deformation. Aifantis $[15,16]$ introduced the Laplacian of the equivalent shear strain and a gradient coefficient into the constitutive equation for the flow stress to represent the scale-dependent deformation localization. The gradient coefficient had to be calibrated using scale-dependent experimental data such as the width of the shear band. These models quantified the localized deformation and reduced the mesh dependence in simulations of nonuniform deformation using discretized numerical methods, such as the finite element method (FEM) [17-19].

Another important gradient theory is the description of the size effect on the mechanical behavior of crystalline materials. In this framework, a plastic nonlocal model based on the dislocation kinetics was constructed. Fleck and Hutchinson [20,21] introduced a geometrically necessary dislocation caused by the strain gradient into models of plastic deformation behavior. Gao et al. [22, 23] proposed a mechanism-based theory of the strain gradient plasticity that linked statistically stored and geometrically necessary dislocations to the plastic strain and strain gradient. Gurtin [24-26] proposed a unique gradient theory by introducing microforces for each slip system that maintained a consistent balance among themselves.

Micro- to macroscopic modeling of nonlocal deformation was also performed using the second-order homogenization method (2nd-HM). In this framework, the scale-dependent deformation behavior of materials with periodic heterogeneous microstructure was evaluated by minimizing the microscopic energy changes caused by both the macroscopic strain and the strain gradient [27, 28]. FEM simulation based on the 2nd-HM enables evaluation of the size-dependent mechanical behavior of any engineering material having microscopic heterogeneity. Computational procedures for the 2nd-HM have been developed in many studies, e.g., generalization of the periodic boundary condition for the representative volume element (RVE) [29, 30], introduction of the microscopic perturbation function for the macroscopic displacement field [31-33], addition of the integral condition on the RVE for the $C^{1}-C^{0}$ continuity transition between the micro- and macroscales for the finite element [34], establishment of the rate-form large-deformation scheme for the 2nd-HM [35, 36], and development of statistical homogenization of random microstructure [37]. Furthermore, the 2nd-HM has been applied to engineering problems, e.g., bending and twisting of thin sheets [38], anisotropic and dispersive wave propagation of heterogeneous materials [39-41], and fracture prediction of brittle materials [42].

A nonlocal model can be developed by comparing the predicted and experimentally quantified strain fields. The author's research group is investigating the interaction of micro- and macroscopic nonuniform deformations of polycrystalline copper. The strain and stress fields during uniaxial tensile tests of a curved specimen in which a stress 
gradient was introduced during testing were quantitatively evaluated using DIC [6, 8]. The key result obtained by this experiment is that the macroscopic strain concentration in specimens with larger crystalline grains is smaller than that in specimens with smaller crystalline grains. This result shows that the resistance to the macroscopic stress gradient may be increased by the nonuniform deformation caused by heterogeneity in the larger microstructure.

To theoretically describe such nonuniform deformation that depends on the microstructure size, it is necessary to establish a framework of the nonlocal mechanical model, into which the experimentally observed strain and stress fields can be incorporated. The author's group proposed using the higher-order stress, which can be evaluated from the stress field obtained experimentally, and formulated the nonlocal constitutive equation, which relates the strain, strain gradient, stress, and higher-order stress [43]. The nonlocal mechanical behavior of elastic material having heterogeneous local strength can be evaluated using the proposed model. The development of a model for the inelastic strain range is important for experiment-based modeling of the size-dependent nonuniform deformation.

The goal of the present study is to establish an experiment-based procedure for modeling the nonlocal properties of engineering materials with microscopic heterogeneity. Many nonlocal models of crystalline materials [20-26] have been were proposed to capture the effect of the absolute size on the mechanical property, e.g., the HallPetch effect. These models are usually based on the dislocation kinetics and can be applied only to crystalline materials. However, to predict the nonuniform deformation of various heterogeneous engineering materials, it is important to evaluate the effect of the relative size at the micro- and macroscales on the nonuniform deformation of the material.

Computational simulation based on the 2nd-HM enables evaluation of the scale-dependent nonuniform deformation of any microscopically heterogeneous material [27-41]. However, care must be taken in such a simulation because the obtained results depend strongly on the choice of RVE. To show the ability of the 2nd-HM to represent the strain field of a heterogeneous material, it is important to compare the simulated and experimentally obtained strain fields. Experimental validation of the strain field in stainless steel having regularly distributed voids has been reported [44]. Comparison of the strain fields in inelastic material having random microstructure may provide an important direction for modeling of the scale-dependent nonuniform deformation of engineering materials.

In this manuscript, the micro- to macroscopic nonuniform deformation of polycrystalline copper is evaluated by a numerical simulation based on the rate-form 2nd-HM. To investigate size-dependent nonuniform deformation, FEM simulations of a mechanical test conducted in an experimental study are performed. The effects of the microstructure size on the macroscopic response and the nonuniform deformation under tensile testing of a polycrystalline copper specimen are investigated by computational simulation. Finally, the accuracy of the 2nd-HM is discussed by comparing the strain field development obtained from the simulation and the experimental results.

\section{FORMULATION OF HIGHER-ORDER CONSTITUTIVE EQUATION}

The higher-order rate form constitutive equation that relates the first- and second-order gradients of the velocity, stress rate, and higher-order stress rate is formulated in this section. Even if the mechanical properties are macroscopically uniform, engineering materials generally exhibit microscopic heterogeneity, which affects their deformation behavior. Now, we consider an elasto-viscoplastic material having the periodically heterogeneous microstructure shown in Fig. 1. First, a local evaluation region of the macrostructure having a finite volume $V$ centered at $x_{i}^{0}$ is defined. The coordinate $x_{i}^{0}$ in this region satisfies the following condition: 


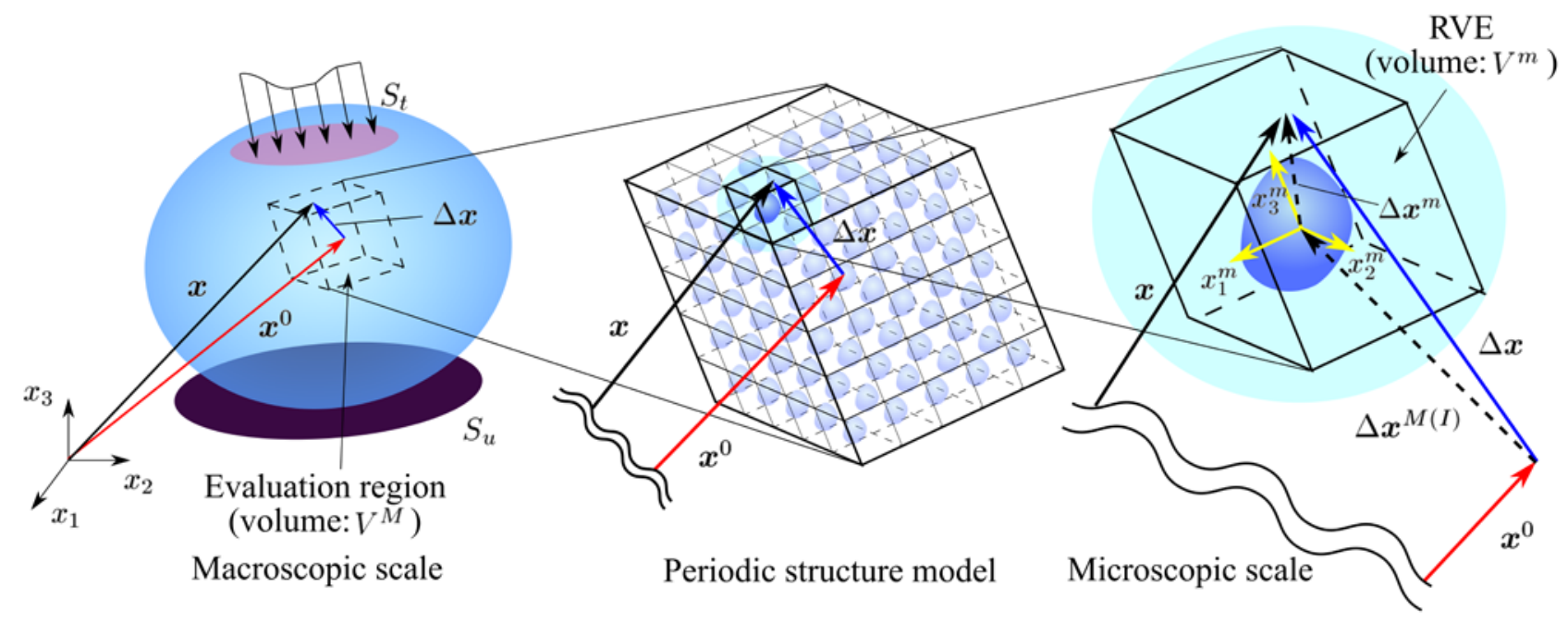

Fig. 1 Schematics of micro- to macroscopic structure

$$
\int_{V}\left(x_{i}-x_{i}^{0}\right) d V=\int_{V} \Delta x_{i} d V=0
$$

Next, we consider the microscopic deformation. The microscopically heterogeneous material is modeled using the periodic structure with a finite volume $Y$. The RVE is chosen from within the microstructure, and the microscopic coordinates of the Ith RVE are defined using the microscopic relative coordinate $\Delta x_{i}^{m}$, which is a periodic coordinate, and the relative center of the Ith RVE $\Delta x_{i}^{M(I)}$ (see Fig. 1). The relationship between the microand macroscopic coordinates is

$$
x_{i}=x_{i}^{0}+\Delta x_{i}=x_{i}^{0}+\Delta x_{i}^{M(I)}+\Delta x_{i}^{m} .
$$

The microscopic relative coordinate also satisfies the following condition:

$$
\int_{Y} \Delta x_{i}^{m} d Y=0
$$

In a way similar to that used in a previously proposed second-order computational homogenization scheme $[27,28]$, the velocity $v_{i}$ is decomposed into the macroscopic velocity $v_{i}^{M}$ and the microscopic velocity fluctuation $v_{i}^{m}$ as

$$
v_{i}=v_{i}^{M}+v_{i}^{m}
$$

The macroscopic velocity $v_{i}^{M}$ in this region can be expressed by second-order Taylor expansion as follows:

$$
v_{i}^{M}=v_{i}^{0}+v_{i, j}^{0} \Delta x_{j}+\frac{1}{2} v_{i, j k}^{0} \Delta x_{j} \Delta x_{k},
$$

where $v_{i}^{0}, v_{i, j}^{0}$ and $v_{i, j k}^{0}$ are the velocity, and the first and second-order gradients of the velocity at the center of the evaluation region, respectively. Note that the parameters with superscript 0 are constant in the evaluation region.

As in the first-order homogenization method, the microscopic velocity fluctuation $v_{i}^{m}$ is given as a function of the macroscopic deformation. To satisfy the continuity of the microscopically periodic deformation under a macroscopic strain and strain gradient, the microscopic fluctuation is separated into two unknown functions, namely, 
those for the macroscopic strain and the macroscopic strain gradient [31-33]. In this study, the unknown function for the constant term is further added to the two functions above to formulate the rate-form elasto-viscoplastic constitutive relationships for the macroscopic field [35, 36], as

$$
v_{i}^{m}=v_{i}^{m 0}+v_{i l m}^{m 1}\left(v_{l, m}^{0}+v_{l, m n}^{0} \Delta x_{n}^{M(I)}\right)+v_{i l m n}^{m 2} v_{l, m n}^{0},
$$

where $v_{i}^{m 0}, v_{i l m}^{m 1}$, and $v_{i l m n}^{m 2}$ are the unknown periodic functions of the constant term and the first and second-order gradients of the macroscopic velocity, respectively. From Eqs. (4) to (6), the velocity gradient is given by

$$
\begin{aligned}
v_{i, j} & =v_{i, j}^{0}+v_{i, j k}^{0} \Delta x_{k}+\frac{\partial v_{i}^{m 0}}{\partial x_{j}^{m}}+\frac{\partial v_{i l m}^{m 1}}{\partial x_{j}^{m}}\left(v_{l, m}^{0}+v_{l, m n}^{0} \Delta x_{n}^{M(I)}\right)+\frac{\partial v_{i l m n}^{m 2}}{\partial x_{j}^{m}} v_{l, m n}^{0} \\
& =\frac{\partial v_{i}^{m 0}}{\partial x_{j}^{m}}+\left\{\delta_{i l} \delta_{j m}+\frac{\partial v_{i l m}^{m 1}}{\partial x_{j}^{m}}\right\}\left(v_{l, m}^{0}+v_{l, m n}^{0} \Delta x_{n}^{M(I)}\right)+\left\{\delta_{i l} \delta_{j m} \Delta x_{n}^{m}+\frac{\partial v_{i l m n}^{m 2}\left(\boldsymbol{x}^{m}\right)}{\partial x_{j}^{m}}\right\} v_{l, m n}^{0} .
\end{aligned}
$$

We consider the elasto-viscoplastic constitutive equation for a material point. In the updated Lagrangian formulation, the relationship between the nominal stress rate $\dot{\pi}_{i j}$ and velocity gradient $v_{i . j}$ is given by

$$
\dot{\pi}_{i j}=\left\{D_{i j k l}-F_{i j k l}+\sigma_{l i} \delta_{j k}\right\} v_{k, l}-P_{i j}=\hat{D}_{i j k l} v_{k, l}-P_{i j},
$$

where $D_{i j k l}$ is the elasto-viscoplastic tangent modulus tensor, $\sigma_{i j}$ is the Cauchy stress, $P_{i j}$ is the viscoplastic term, and $F_{i j k l}=\left\{\sigma_{l j} \delta_{k i}+\sigma_{k j} \delta_{l i}+\sigma_{l i} \delta_{k j}+\sigma_{k i} \delta_{l j}\right\} / 2$.

The unknown periodic functions $v_{i}^{m 0}, v_{i l m}^{m 1}$, and $v_{i l m n}^{m 2}$ can be obtained by solving the microscopic boundary problem (see Appendix A). For simplicity, the microscopic velocity gradient and nominal stress rate are rewritten as

$$
\begin{aligned}
& v_{i, j}=\eta_{i j}^{m 0}+\eta_{i j l m}^{m 1} v_{l, m}^{0}+\left\{\eta_{i j l m}^{m 1} \Delta x_{n}^{M(I)}+\eta_{i j l m n}^{m 2}\right\} v_{l, m n}^{0} . \\
& \dot{\pi}_{i j}=\hat{D}_{i j p q}\left[\eta_{p q}^{m 0}+\eta_{p q r s}^{m 1} v_{r, s}^{0}+\left\{\eta_{p q r s}^{m 1} \Delta x_{t}^{m 0(I)}+\eta_{p q r s t}^{m 2}\right\} v_{r, s t}^{0}\right]-P_{i j},
\end{aligned}
$$

where $\eta_{i j}^{m 0}=\partial v_{i}^{m 0} / \partial x_{j}^{m}, \quad \eta_{i j l m}^{m 1}=\delta_{i l} \delta_{j m}+\partial v_{i l m}^{m 1} / \partial x_{j}^{m}$, and $\eta_{i j l m n}^{m 2}=\delta_{i l} \delta_{j m} \Delta x_{n}^{m}+\partial v_{i l m n}^{m 2} / \partial x_{j}^{m}$.

Subsequently, we obtain the virtual work of the macroscopic evaluation region. The microscopic virtual velocity gradient associated with the macroscopic virtual strain and strain gradient is given by

$$
\delta v_{i, j}=\eta_{i j l m}^{m 1} \delta v_{l, m}^{0}+\left\{\eta_{i j l m}^{m 1} \Delta x_{n}^{M(I)}+\eta_{i j l m n}^{m 2}\right\} \delta v_{l, m n}^{0} .
$$

The virtual work of the macroscopic evaluation region can be obtained as follows.

$$
\frac{\delta W}{V}=\left(\delta v_{i, j}^{0} \delta v_{i, j n}^{0}\right)\left(\left[\begin{array}{cc}
\bar{D}_{i j k l}^{(11)} & \bar{D}_{i j k l m}^{(12)} \\
\bar{D}_{n i j k l}^{(21)} & \bar{D}_{i j k l}^{(11)}\left(J_{n m}-J_{n m}^{m}\right)+\bar{D}_{n i j k l m}^{(22)}
\end{array}\right]\left\{\begin{array}{c}
v_{k, l}^{0} \\
v_{k, l m}^{0}
\end{array}\right\}-\left\{\begin{array}{l}
\bar{P}_{i j}^{(1)} \\
\bar{P}_{n i j}^{(2)}
\end{array}\right\}\right),
$$

where $\quad \bar{D}_{i j k l}^{(11)}=\int_{Y} \eta_{p q i j}^{m 1} \hat{D}_{p q r s} \eta_{r s k l}^{m 1} d Y / Y \quad, \quad \bar{D}_{i j k l m}^{(12)}=\int_{Y} \eta_{p q i j}^{m 1} \hat{D}_{p q r s} \eta_{r s k l m}^{m 2} d Y / Y \quad, \quad \bar{D}_{n i j k l}^{(21)}=\int_{Y} \eta_{p q i j n}^{m 2} \hat{D}_{p q r s} \eta_{r s k l}^{m 1} d Y / Y$, $\bar{D}_{n i j k l m}^{(22)}=\int_{Y} \eta_{p q i j n}^{m 2} \hat{D}_{p q r s} \eta_{r s k l m}^{m 2} d Y / Y, \quad \bar{P}_{i j}^{(1)}=\int_{Y} \eta_{p q i j}^{m 1}\left\{P_{p q}-\hat{D}_{p q r s} \eta_{r s}^{m 0}\right\} d Y / Y, \quad \bar{P}_{n i j}^{(2)}=\int_{Y} \eta_{p q i j n}^{m 2}\left\{P_{p q}-\hat{D}_{p q r s} \eta_{r s}^{m 0}\right\} d Y / Y$, and $J_{i j}=\frac{1}{V} \int_{V} \Delta x_{i} \Delta x_{j} d V, \quad J_{i j}^{m}=\frac{1}{Y} \int_{Y} \Delta x_{i}^{m} \Delta x_{j}^{m} d Y$. Eq. (12) is derived in Appendix B.

The rates of nominal stress $\dot{\pi}_{i j}^{0}$ and higher-order nominal stress $\dot{\tau}_{n i j}^{0}$ in the macroscopic evaluation region are defined as the quantities work conjugate to $\delta v_{i, j}^{0}$ and $\delta v_{i, j k}^{0}$ respectively. Therefore, the higher-order 
constitutive equation for the macroscopic evaluation region having periodic microstructure is given by

$$
\left\{\begin{array}{c}
\dot{\pi}_{i j}^{0} \\
\dot{\tau}_{n i j}^{0}
\end{array}\right\}=\left[\begin{array}{cc}
\bar{D}_{i j k l}^{(11)} & \bar{D}_{i j k l m}^{(12)} \\
\bar{D}_{n i j k l}^{(21)} & \bar{D}_{i j k l}^{(11)}\left(J_{n m}-J_{n m}^{m}\right)+\bar{D}_{n j k k l m}^{(22)}
\end{array}\right]\left\{\begin{array}{c}
v_{k, l}^{0} \\
v_{k, l m}^{0}
\end{array}\right\}-\left\{\begin{array}{c}
\bar{P}_{i j}^{(1)} \\
\bar{P}_{n i j}^{(2)}
\end{array}\right\} .
$$

The macroscopic boundary problem can be solved using the macroscopic virtual work for the evaluation region.

\section{COMPUTATIONAL SIMULATION}

\subsection{Simulation Model}

To represent the elasto-viscoplastic deformation of the microscopic crystalline structure, we employed the classical crystalline plasticity theory [45], in which the rate-form constitutive equation is given by

$$
\stackrel{\nabla}{S_{i j}}=D_{i j k l} d_{k l}-\sum_{(\alpha)} R_{i j}^{(\alpha)} \dot{\gamma}^{(\alpha)},
$$

where $\stackrel{\nabla}{S}_{i j}$ is the Jaumann rate of the Kirchhoff stress, $d_{i j}$ is the strain rate tensor, $R_{i j}^{(\alpha)}=D_{i j k l} P_{k l}^{(\alpha)}$ $+W_{i k}^{(\alpha)} \sigma_{k j}-\sigma_{i k} W_{k j}^{(\alpha)}, \quad P_{i j}^{(\alpha)}=\left(s_{i}^{*(\alpha)} m_{j}^{*(\alpha)}+s_{j}^{*(\alpha)} m_{i}^{*(\alpha)}\right) / 2$ is the Schmid tensor, $W_{i j}^{(\alpha)}=\left(s_{i}^{*(\alpha)} m_{j}^{*(\alpha)}-s_{j}^{*(\alpha)} m_{i}^{*(\alpha)}\right) / 2$, $s_{i}^{*(\alpha)}=F_{i j}^{*} s_{j}^{(\alpha)}, m_{i}^{*(\alpha)}=m_{i}^{(\alpha)} F_{i j}^{*-1}, F_{i j}^{*}$ is the deformation gradient for elastic deformation and rotation, and $s_{i}^{(\alpha)}$ and $m_{i}^{(\alpha)}$ are unit vectors in the slip direction and normal to the slip plane of the $\alpha$ slip system, respectively. The equivalent shear strain rate $\dot{\gamma}^{(\alpha)}$ is given by a power law [46] as

$$
\dot{\gamma}^{(\alpha)}=\dot{\gamma}_{0} \operatorname{sgn}\left(\tau^{(\alpha)}\right)\left(\frac{\tau^{(\alpha)}}{g^{(\alpha)}}\right)^{\frac{1}{m}},
$$

where $\tau^{(\alpha)}=P_{i j}^{(\alpha)} \sigma_{i j}$ is the shear stress on the slip plane, and $\dot{\gamma}_{0}$ and $m$ are material constants. Further, $g^{(\alpha)}$ is the resistance to the slip, which is given by following equation;

$$
\dot{g}^{(\alpha)}=\sum_{(\beta)}\{q h+(1-q) h\} \delta_{\alpha \beta}\left|\dot{\gamma}^{(\beta)}\right|,
$$

where $h=h_{0} \operatorname{sech}\left\{h_{0} \gamma /\left(\tau_{s}-\tau_{0}\right)\right\} ; q, h_{0}, \tau_{s}$, and $\tau_{0}$ are material constants; and $\gamma$ is the sum of the slip for all slip systems. As mentioned, the objective of this study is to evaluate the effect of the relative size caused by interaction between micro- and macroscopic nonuniform deformation. To eliminate the effect of the absolute-size in a crystalline material, the size-independent hardening equation is employed in this study. The material parameters employed in this study are shown in Table 1.

The author's research group quantitatively evaluated the strain field on a polycrystalline copper specimen under tensile deformation associated with a stress gradient. In this experiment, a tensile load was applied to a specimen having a curved gage section, and the effects of the curvature and grain size on the nonuniform deformation were discussed [6, 8]. These studies revealed that the strain concentration caused by the macroscopic stress gradient decreased with increasing grain size because the microscopic strain was localized owing to the anisotropic strength distribution of the coarse grains.

Table 1 Material parameters for the crystalline plasticity constitutive equation

\begin{tabular}{cccccc}
\hline$m$ & $\dot{\gamma}_{0}$ & $\tau_{0}$ & $\tau_{s}$ & $h_{0}$ & $q$ \\
\hline 0.05 & $0.001 \mathrm{~s}^{-1}$ & $15 \mathrm{MPa}$ & $55 \mathrm{MPa}$ & $250 \mathrm{MPa}$ & 1.2 \\
\hline
\end{tabular}




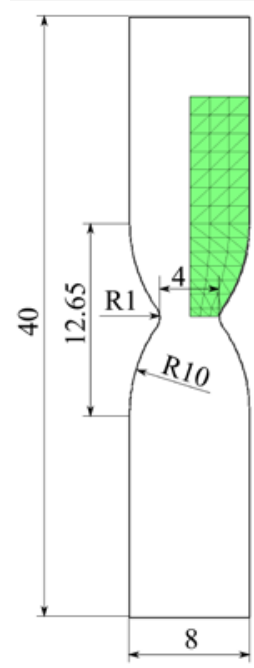

Specimen R

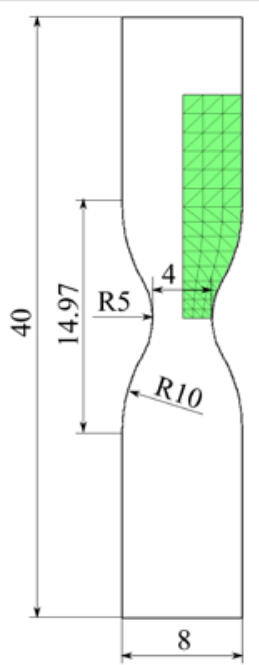

Specimen R5

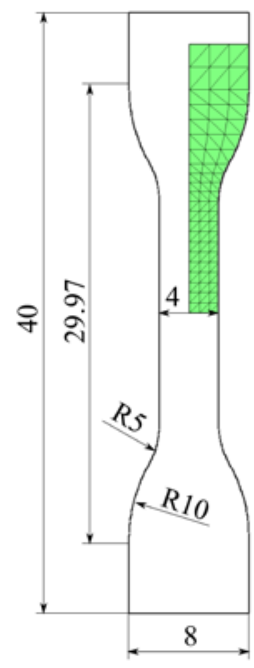

Specimen Rinf

(a) Computational model of macrostructure [in $\mathrm{mm}$ ]

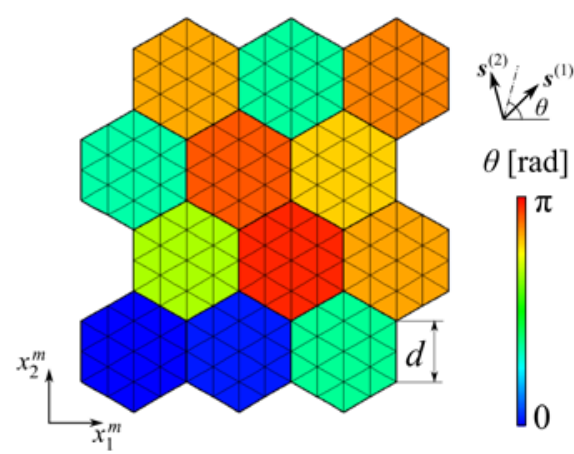

(b) Computational model for microstructure

Fig. 2 Micro- to macroscopic computational models for 2nd-HM

To investigate this size-dependent nonuniform deformation, FEM simulations of the mechanical test conducted in the experimental study are performed.

Fig. 2(a) shows the shapes of specimens with different curvatures in the gage section. The minimum cross-section and the curvature of the shoulder area were kept constant, whereas the length and curvature of the gage section were different for each specimen. Specimens with curvature radii of 1 and $5 \mathrm{~mm}$ and a specimen without curvature were prepared and are referred to hereafter as specimens R1, R5, and Rinf, respectively. A constant tensile velocity of $V=1 \mathrm{~mm} / \mathrm{s}$ was applied to each specimen. The macrostructure was meshed using a six-node second-order triangular element with an integration point. The macroscopic strain gradient of the element could thus be estimated from the nodal displacements.

The polycrystalline microstructure illustrated in Fig. 2 (b) was applied to all the integration points of the macrostructure. The polycrystalline structure employed in this study consisted of grains with uniform size and shape, to which random crystalline orientations were applied. A plane strain two-slip system model [45] was applied to represent the slip deformation of the two-dimensional polycrystalline structure. This model is a simplified model of in-plane slip deformation of a FCC metal. For each grain, 24 second-order finite elements were used, and the separation and slip at the grain boundary were constrained. Therefore, nonuniform deformation occurs through the grain boundaries owing to the differences in crystalline orientation between adjacent grains. When the macroscopic strain gradient is not applied to the microstructure, the nonuniform deformation in the RVE is independent of the RVE size. However, when the macroscopic strain gradient is given, the RVE size affects the nonuniform deformation of the polycrystalline structure because the change in the shape of the RVE owing to the macroscopic strain gradient depends on its relative size. To investigate the effect of the microstructure size, microstructures with different scales, $d=1,10$, and $100 \mu \mathrm{m}$ were prepared.

Note that the mechanical conditions modeled in the simulation are not exactly the same as the experimental conditions. The plastic deformation of the crystal grain to limited in the plane, and the heterogeneity in thickness direction cannot be incorporated into this model. Therefore, it is difficult to directly compare the quantitative results for the mechanical response and strain field. However, the size effect on the nonuniform deformation of the polycrystalline 
material can be evaluated from the simulation results for different microstructure sizes and different macroscopic stress gradients. We discuss the characteristics of nonlocal deformation of a material with microscopic heterogeneity by comparing the size effects obtained in the simulation with those observed in the experiment.

\subsection{Simulation Results}

This section presents computational results obtained from FEM simulations based on the 2nd-HM. Fig. 3 shows the relationships between the tensile load divided by the minimum cross-section and the tensile displacement for specimens R1, R5, and Rinf for different grain sizes. Because the size of the gage section is different for each specimen, the increase in the tensile load due to the applied displacement depends on the curvature radius. A specimen with a smaller curvature radius shows a larger maximum stress even though the minimum cross-section is the same for different specimens. The reasons are that i) the multiaxial stress increases at the gage section for specimens with a smaller curvature, ii) bending deformation, in addition to tensile deformation, increases with decreasing curvature radius, and iii) the decrease in thickness associated with strain concentration is constrained owing to the plane strain condition.

Results for different microstructure sizes are plotted in different colors. The responses of the Rinf specimen for different microstructure sizes are similar. By contrast, the tensile loads for specimens R1 and R5 with $d=100$ are slightly smaller than those for the specimens with smaller grains. When the macroscopic strain gradient is given to the microstructure, the difference in the macroscopic strain is enlarged by increasing microstructure size. The decreasing tensile load for the larger grain specimens indicates that the nonuniform deformation enhanced by the macroscopic strain gradient decreased the resistance to the tensile deformation. The microscopic strain field is described later in this section.

In actual polycrystalline metals, an apparent size effect on the macroscopic response usually appears as the HallPetch effect, which is related to the dislocation kinetics at more microscopic scales. The computational results indicate that the interaction between nonuniform deformation at the micro- and macroscopic scales also induces a slight size effect on the response of the material.

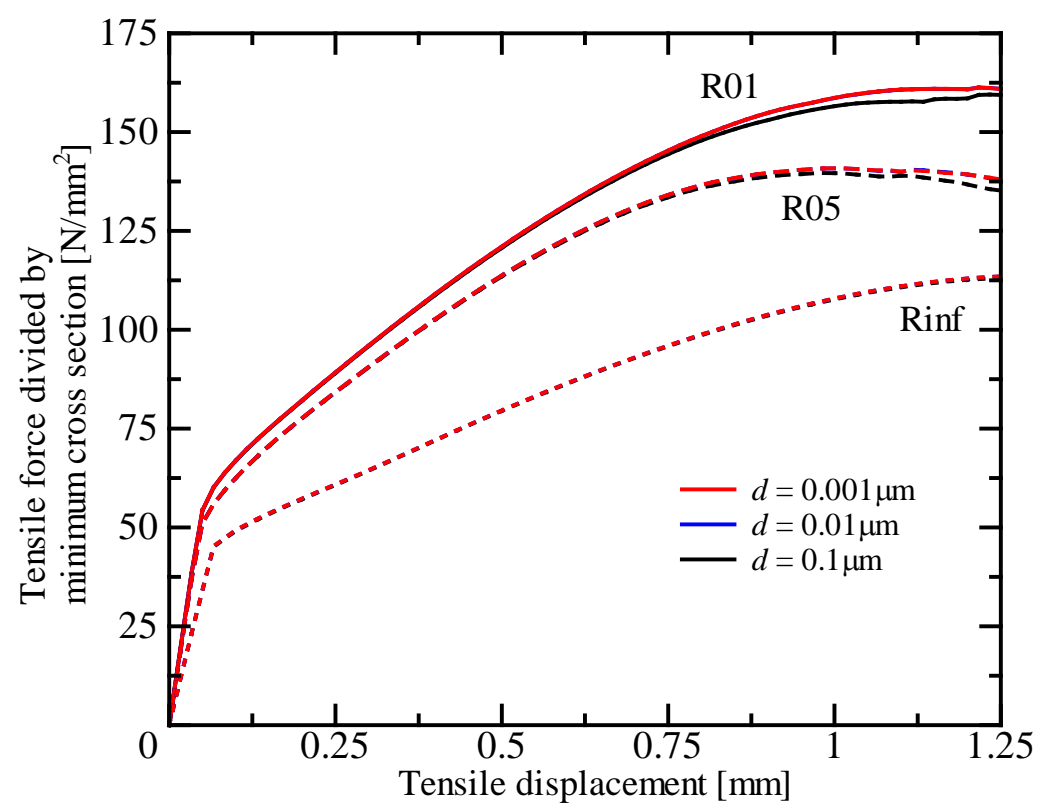

Fig. 3 Relationships between tensile force divided by minimum cross-section and tensile displacement 

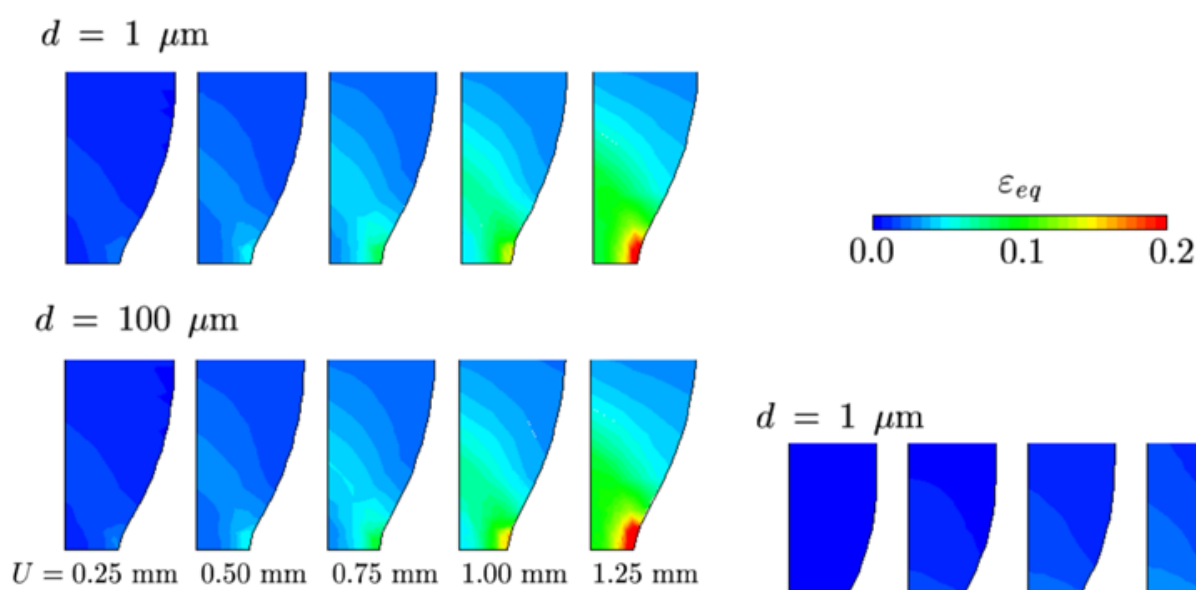

(a) Specimen R1

$d=1 \mu \mathrm{m}$

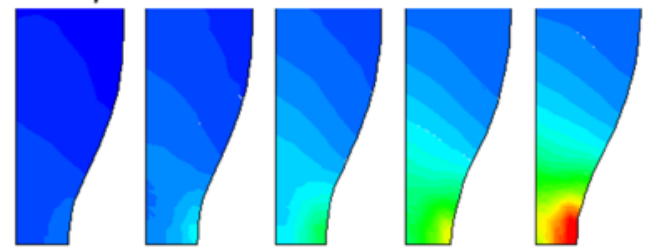

$d=100 \mu \mathrm{m}$

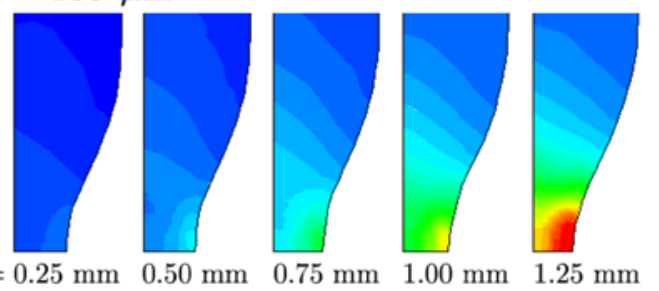

(b) Specimen R5
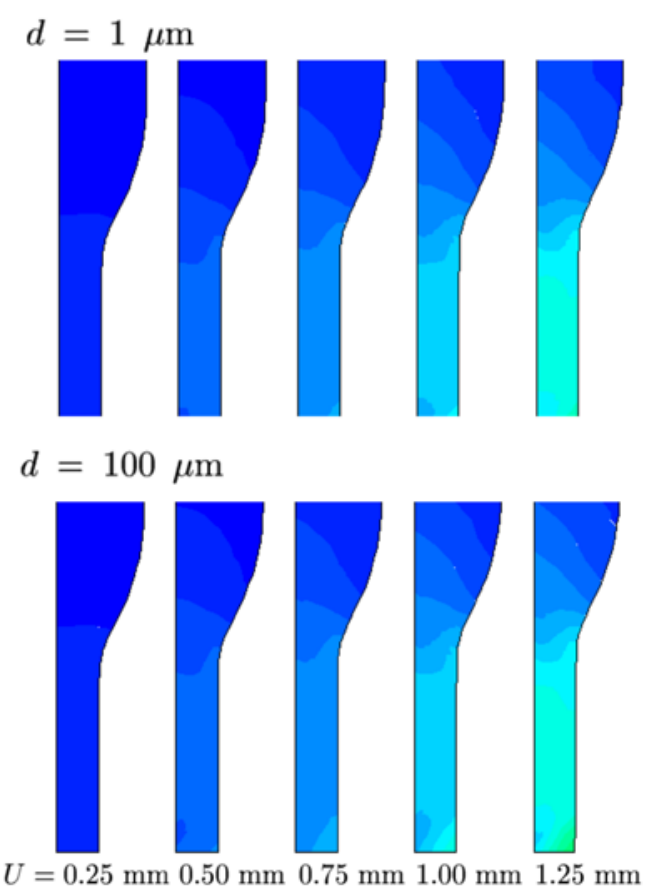

(c) Specimen Rinf

Fig. 4 Development of equivalent strain distribution in the macrostructure

Fig. 4 presents the development of the macroscopic strain field. Obvious strain concentration is observed in specimens R1 and R5. In specimen R1, an inclined deformation band develops in the curved area; however, the deformation band tends to occur in the direction transverse to the load. Almost uniform strain occurs in specimen Rinf. These differences in the development of the strain field affect the increase in the tensile load as shown in Fig. 3. As shown in the figure, the microstructure size has little effect on the macroscopic strain distribution. Although the magnitude of the strain increases with the microstructure size, the distribution patterns are similar for specimens with different microstructure sizes.

Fig. 5 illustrates the development of the strain field in the microstructure allocated at macroscopically strain concentrated area (Point A in Fig. 5) for specimens R1 and R5. The nonuniform deformation accompanying the microscopic shear band is due to the anisotropy of the grains. Although the crystalline orientation pattern of the microstructure is the same, the development of the strain distribution depends on the microstructure size when a larger macroscopic strain gradient is applied to the microstructure. The corresponding periodic boundaries are almost parallel for the microstructures with $d=1 \mu \mathrm{m}$, whereas they become divergent owing to the macroscopic strain gradient for the microstructures with $d=$ $100 \mu \mathrm{m}$. This effect enhances the nonuniformity of the deformation slightly, and it results in a decrease in the tensile load for the specimens with the larger grains. 


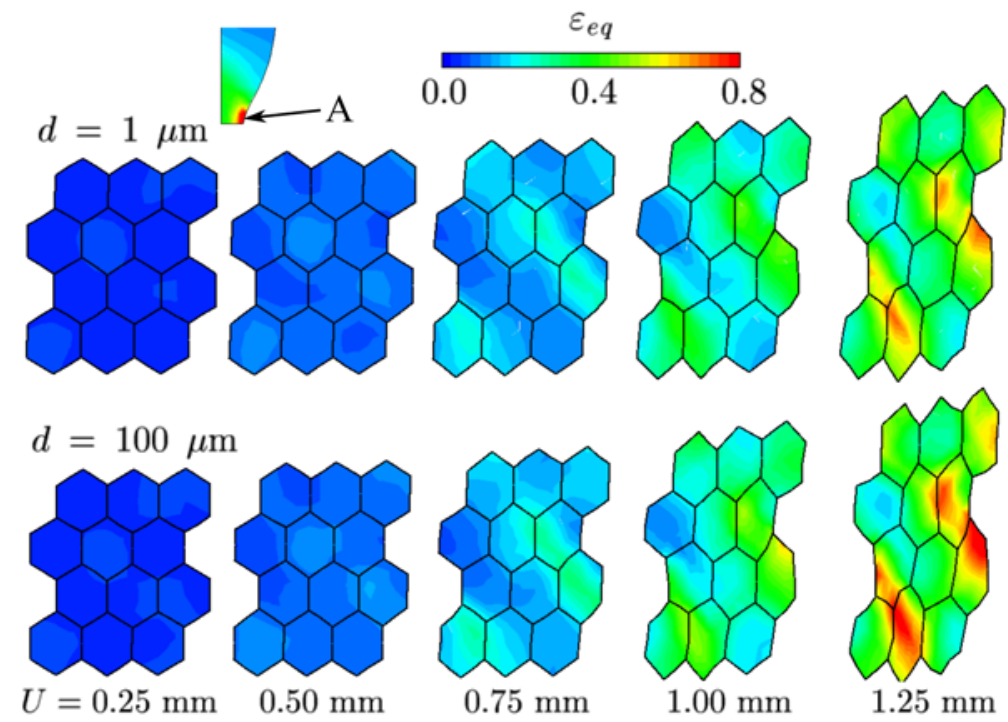

(a) Specimen R1
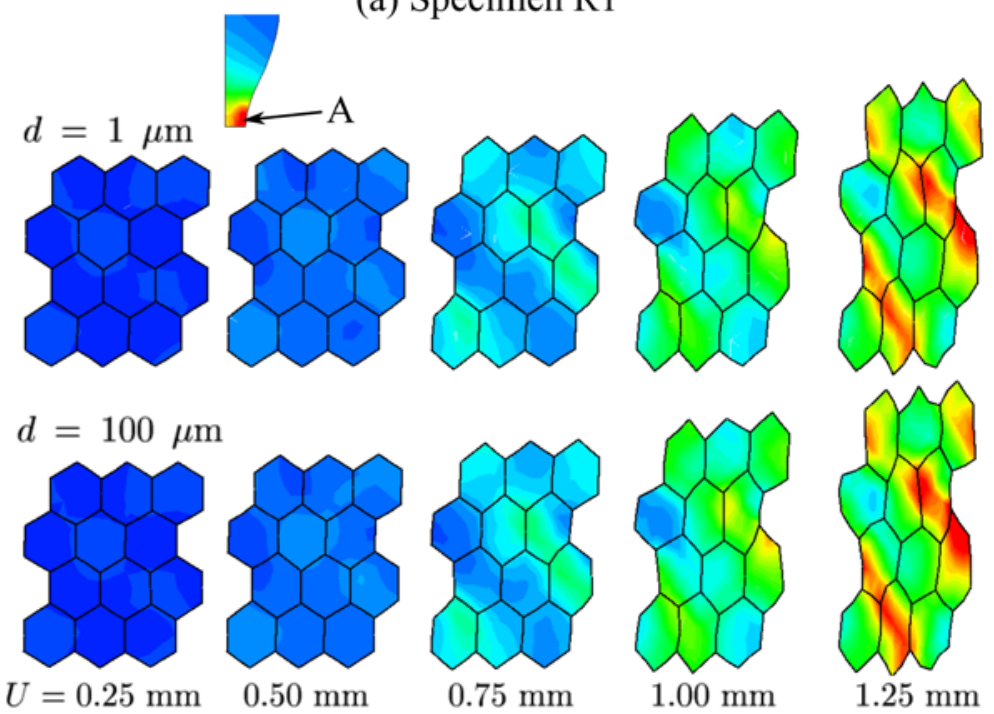

(b) Specimen R5

Fig. 5 Development of equivalent strain distributions in the microstructure

\section{DISCUSSION}

The interaction between micro- and macroscopic nonuniform deformation of polycrystalline copper was numerically investigated in Section 3. As mentioned above, the author's research group also evaluated the nonuniform deformation of pure copper experimentally [6, 8]. In the experimental studies, a development of the strain field on the surface of specimens having the same shapes as those in the FEM simulations was quantitatively evaluated using DIC. In this section, the nonuniform deformation of polycrystalline copper is investigated by comparing the simulated and experimental strain field results. Fig. 6 shows the distributions of the equivalent strain obtained by the FEM simulation and the corresponding experiment for (a) specimen R1 with small grains, (b) specimen R1 with large grains, (c) specimen R5 with small grains, and (d) specimen R5 with large grains. The specimens with small and large grains used in the experimental study were obtained at the annealing temperatures of 450 and $850{ }^{\circ} \mathrm{C}$, and the average grain sizes were approximately 27 and $121 \mu \mathrm{m}$, respectively.

As shown in Figs. 6 (a) and (c), the simulated and experimental strain fields in specimens with small grains are 


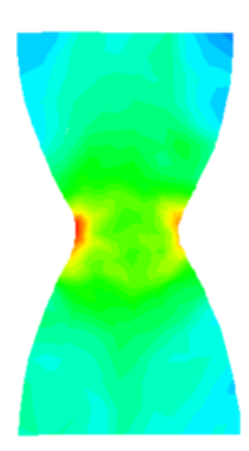

Experimental result

(a) Specimen R1 with smaller grains

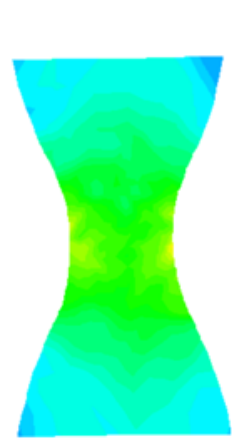

Experimental result

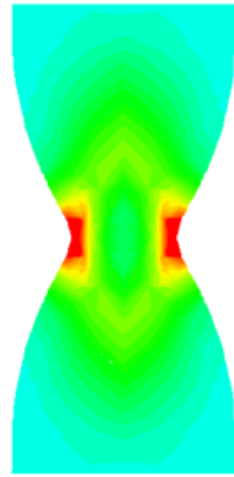

Simulation result

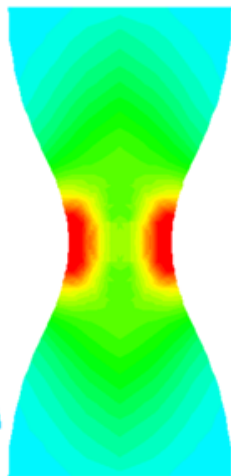

Simulation result
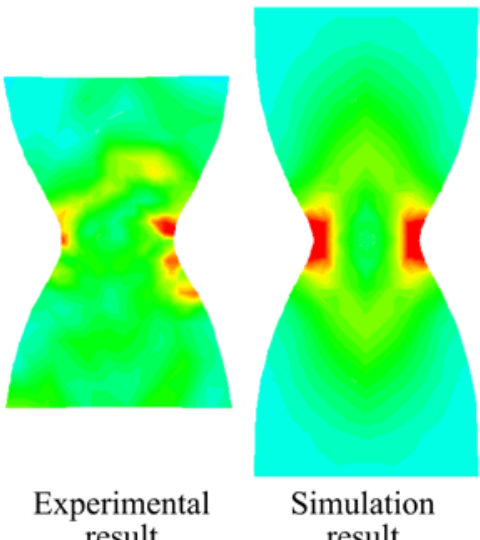

Simulation result

(b) Specimen R1 with larger grains

(c) Specimen R5 with smaller grains
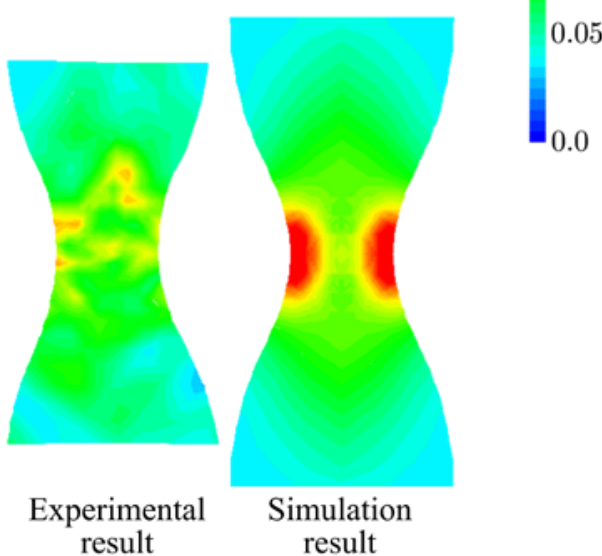

(d) Specimen R5 with larger grains

Fig. 6 Comparison of simulated and experimentally observed equivalent strain distributions

similar except for the magnitude of the strain. However, the strain field evaluated by DIC for specimens with large grains (Figs. 6 (b) and (d)) are quite different from those obtained from the FEM simulation. Random strain localization owing to the anisotropic strength distribution of coarse grains can be observed in the experimental results for specimens with large grains, whereas the simulation shows that the strain field following the macroscopic stress gradient.

In the FEM simulation based on the 2nd-HM, we can evaluate the effect of heterogeneous microstructure with a specific size on the nonuniform deformation of the macrostructure. The effect of the microstructure size is greater when the size difference between the micro- and macrostructures is small. However, because the macrostructure in the homogenization method is assumed as a homogeneous material having the periodic heterogeneous microstructure, the randomly distributed strain localization caused by the aperiodicity of the microstructure, which is also observed when the size difference is small, may not be apparent. If different microstructures are assigned to different macroscopic integration points to represent the randomness of the material strength, the simulation result depends strongly on the mesh division of the macrostructure.

Here we discuss the strain field in the specimen further. Fig. 7 presents the development of the equivalent strain distribution in specimen R1 around the evaluation points along the line A-A' in Fig. 7. The simulated and experimental strain fields for the specimens with smaller and larger grains are compared. Although both the experimental and simulation results show a strain concentration around the minimum cross-sectional area, the magnitude of the concentration in the 

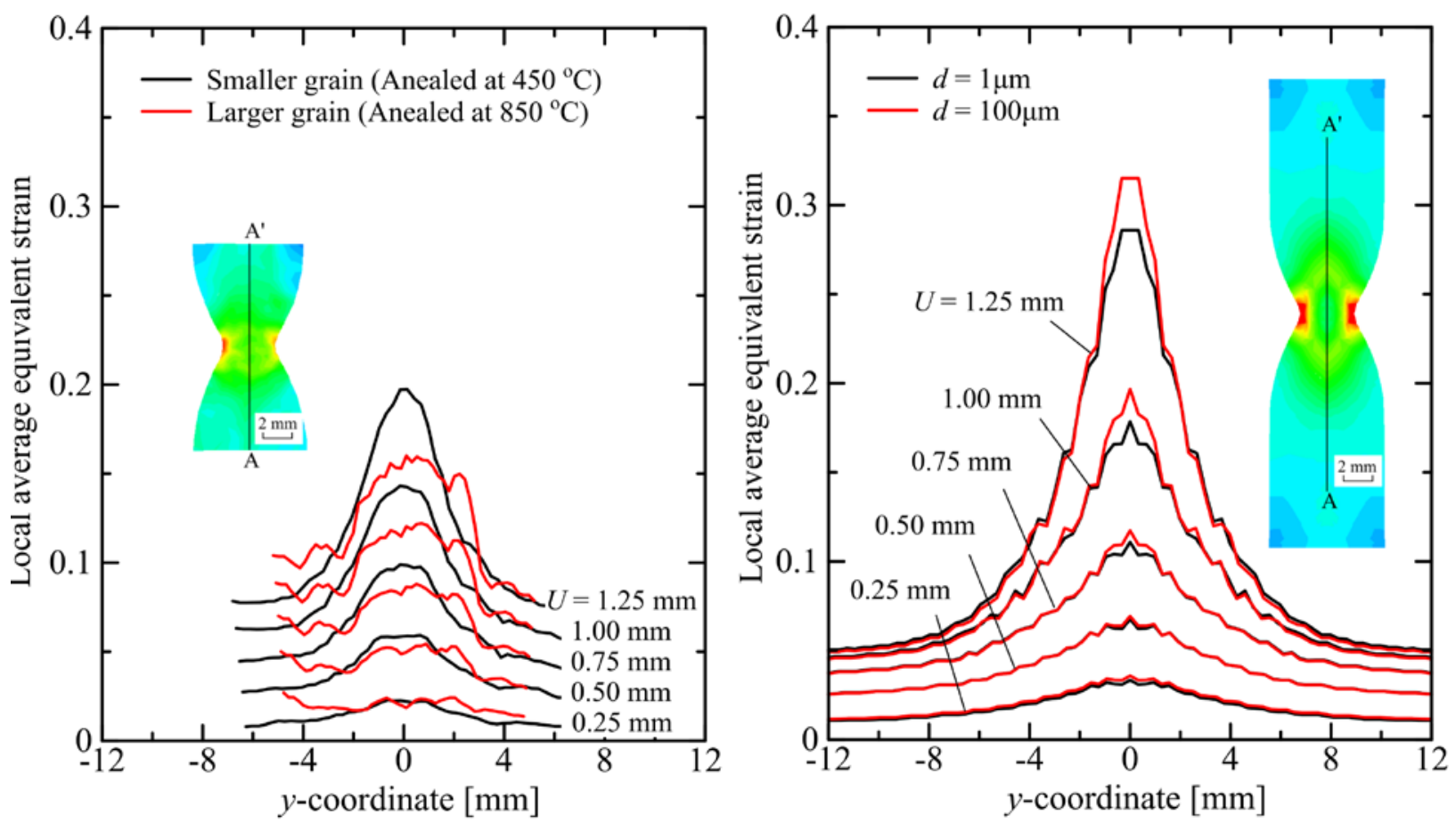

Fig. 7 Comparison of the equivalent strain distribution on line A-A'

experimental result is smaller than that in the simulation result. In the experiment, random strain localization owing to the microscopic heterogeneity occurs independent of the macroscopic stress gradient, and it decreases the strain concentration around the minimum cross-sectional area. The local strain gradient is increased by random strain localization in the experimental results for the specimens with large grains. In the FEM simulation, a steeper strain gradient is also observed for the specimen with $d=100 \mu \mathrm{m}$. However, the decrease in strain concentration with the macroscopic stress gradient cannot be represented because random strain localization caused by microscopic heterogeneity does not occur in the FEM simulation based on the 2nd-HM.

A series of computational results revealed that the local strain gradient is enhanced because the local resistance to the macroscopic strain gradient decreases with increasing crystalline grain size, as observed in the experimental study. This result provides important information for predicting the localization of the strain in engineering structures. In the experimental result, however, the randomness of the microstructure caused many strain localization sites, which decreased the global strain gradient caused by the macroscopic stress gradient. The FEM model could not represent this random strain distribution because a periodic microstructure was employed in the homogenization scheme.

Therefore, although the 2nd-HM may be able to evaluate the scale-dependent nonuniform deformation of materials having regular periodic microstructure [44], it is difficult to represent the development of the nonuniform deformation in terms of the microstructure size when the material has highly random microstructure. The introduction of discontinuous randomness in the material into the macroscopic simulation model is a possible strategy. However, it is unrealistic to construct a full-scale simulation model of a large structure with microscopic randomness.

An important characteristic indicated by the comparison of the simulation and experimental results is the difference in the direction of the stress and strain gradients. In the simulation result, when the directions coincide, the strain gradient is enhanced by the stress gradient. However, the strain gradient in the stress gradient direction is decreased when the 
directions are different owing to the randomness of the microstructure in the actual material. This indicates that engineering materials with microscopic heterogeneity have an intrinsic nonlocal strength corresponding to the randomness of the microstructure. Quantitative evaluation of the effect of the randomness of the microstructure on the relationship between the stress and strain gradients may characterize this nonlocal strength of the material.

\section{CONCLUSION}

The effect of the size difference between the micro and macroscopic scales on the nonuniform deformation of a polycrystalline material was numerically investigated using the rate-form 2nd-HM. FEM simulations of tensile tests of polycrystalline pure copper specimens having curved gage sections with different curvatures and different grain sizes, which correspond to the experimental studies, were performed.

When macroscopic nonuniform deformation accompanied by a stress gradient occurred in the curved gage section of the specimens with large crystalline grains, the macroscopic tensile load decreased slightly compared to that for the specimens with small grains. This decrease in tensile load is related to the nonuniform deformation of the polycrystalline microstructure, which was slightly enhanced by the macroscopic strain gradient in the larger microstructure. The results indicated that the interaction between the nonuniform deformation at the micro- and macroscopic scales induces a slight size effect on the mechanical response.

The nonuniform deformation obtained in the FEM simulation was compared to that evaluated experimentally. The simulated and experimental strain fields for the specimens with small grains were similar, whereas random strain localization was observed in the specimens with large grains only in the experimental study. This random strain localization was caused by the anisotropic strength distribution of coarse grains, which could not be represented in the macroscopic simulation model of the 2nd-HM. Furthermore, the experimental results showed that the strain concentration accompanied by the stress gradient in the curved gage section of the specimen was decreased by the random strain localization owing to the microscopic heterogeneity. However, the strain concentration around the gage section was enhance in the FEM simulation because random strain localization did not occur in the macrostructure.

Therefore, although the 2nd-HM may be able to evaluate the scale-dependent nonuniform deformation of material having regular periodic microstructure, it is difficult to represent the development of nonuniform deformation in terms of the microstructure size when the microstructure of material is highly random. Quantitative evaluation of the effect of the randomness of the microstructure on the relationship between the stress and strain gradients may determine this nonlocal strength of the material.

\section{ACKNOWLEDGEMENTS}

Authors gratefully acknowledge support from JSPS Grants-in-Aid for Scientific Research, Grant Numbers 18K03844.

\section{Author contribution statement}

M. Uchida have made the draft of the manuscript. All authors have read and approved the final manuscript.

\section{Appendix A: Boundary problem for microstructure}


We estimate three unknown functions $v_{i}^{m 0}, v_{i l m}^{m 1}$, and $v_{i l m n}^{m 2}$ by minimizing the microscopic virtual work. The virtual work of the Ith RVE for an arbitrary microscopic deformation is given by

$$
\delta W^{m(I)}=\int_{Y} \delta v_{i, j} \dot{\pi}_{i j} d Y .
$$

By substituting Eqs (7) and (8) into Eq. (A.1), we obtain

$$
\begin{aligned}
& \delta W^{m(I)}=\int_{Y} \delta v_{i, j}\left\{\hat{D}_{i j k l} \frac{\partial v_{i}^{m 0}}{\partial x_{j}^{m}}-P_{i j}\right\} d Y \\
&+\int_{Y} \delta v_{i, j} \hat{D}_{i j k l}\left\{\delta_{i l} \delta_{j m}+\frac{\partial v_{i l m}^{m 1}}{\partial x_{j}^{m}}\right\} d Y\left(v_{l, m}^{0}+v_{l, m n}^{0} x_{n}^{M(I)}\right) \\
&+\int_{Y} \delta v_{i, j} \hat{D}_{i j k l}\left\{\delta_{i l} \delta_{j m} \Delta x_{n}^{m}+\frac{\partial v_{i l m n}^{m 2}}{\partial x_{j}^{m}}\right\} d Y v_{l, m n}^{0} .
\end{aligned}
$$

Because the microscopic fluctuation is assumed to be periodic, the three unknown functions should be obtained without defining the macroscopic deformation and the location of the RVE center. Therefore, three unknown functions minimizing the virtual work of arbitrary micro- and macroscopic deformation states can be obtained by solving the following equations under a periodic condition for RVE.

$$
\begin{aligned}
& \int_{Y} \delta v_{i, j}\left\{\hat{D}_{i j k l} \frac{\partial v_{i}^{m 0}}{\partial x_{j}^{m}}-P_{i j}\right\} d Y=0, \\
& \int_{Y} \delta v_{i, j} \hat{D}_{i j k l}\left\{\delta_{i l} \delta_{j m}+\frac{\partial v_{i l m}^{m 1}}{\partial x_{j}^{m}}\right\} d Y=0, \\
& \int_{Y} \delta v_{i, j} \hat{D}_{i j k l}\left\{\delta_{i l} \delta_{j m} \Delta x_{n}^{m}+\frac{\partial v_{i l m n}^{m 2}}{\partial x_{j}^{m}}\right\} d Y=0 .
\end{aligned}
$$

Solving processes of $v_{i}^{m 0}$ and $v_{i l m}^{m 1}$ are similar to the first-order homogenization method based on the updated Lagrangian formulation. As shown in Eqs. (A.3) to (A.5), the three unknown functions describing the microscopic velocity fluctuation can be obtained only in the RVE.

\section{Appendix B: Formulation of macroscopic constitutive equation}

The macroscopic constitutive equation is formulated using the microscopic velocity fluctuation functions, $\eta_{i j}^{m 0}, \eta_{i j l m}^{m 1}$, and $\eta_{i j l m n}^{m 2}$. The microscopic virtual velocity gradient associated with the macroscopic virtual strain and strain gradient is given by

$$
\delta v_{i, j}=\eta_{i j l m}^{m 1} \delta v_{l, m}^{0}+\left\{\eta_{i j l m}^{m 1} \Delta x_{n}^{M(I)}+\eta_{i j l m n}^{m 2}\right\} \delta v_{l, m n}^{0} .
$$

Therefore, the virtual work of the Ith RVE is calculated as 


$$
\frac{\delta W^{m(I)}}{Y}=\left(\begin{array}{ll}
\delta v_{i, j}^{0} & \delta v_{i, j n}^{0}
\end{array}\right)\left(\left[\begin{array}{cc}
\bar{D}_{i j k l}^{(11)} & \bar{D}_{i j k l}^{(11)} \Delta x_{m}^{M(I)}+\bar{D}_{i j k l m}^{(12)} \\
\Delta x_{n}^{M(I)} \bar{D}_{i j k l}^{(11)}+\bar{D}_{n i j k l}^{(21)} & \Delta x_{n}^{M(I)} \bar{D}_{i j k l}^{(11)} \Delta x_{m}^{M(I)}+\Delta x_{n}^{M(I)} \bar{D}_{i j k l m}^{(12)} \\
+\bar{D}_{n i j k l}^{(21)} \Delta x_{m}^{M(I)}+\bar{D}_{n i j k l m}^{(22)}
\end{array}\right]\left\{\begin{array}{c}
v_{k, l}^{0} \\
v_{k, l m}^{0}
\end{array}\right\}-\left\{\begin{array}{c}
\bar{P}_{i j}^{(1)} \\
\bar{P}_{n i j}^{(2)}
\end{array}\right\}\right),
$$

where $\quad \bar{D}_{i j k l}^{(11)}=\int_{Y} \eta_{p q i j}^{m 1} \hat{D}_{p q r s} \eta_{r s k l}^{m 1} d Y / Y \quad, \quad \bar{D}_{i j k l m}^{(12)}=\int_{Y} \eta_{p q i j}^{m 1} \hat{D}_{p q r s} \eta_{r s k l m}^{m 2} d Y / Y \quad, \quad \bar{D}_{n i j k l}^{(21)}=\int_{Y} \eta_{p q i j n}^{m 2} \hat{D}_{p q r s} \eta_{r s k l}^{m 1} d Y / Y$, $\bar{D}_{n i j k l m}^{(22)}=\int_{Y} \eta_{p q i j n}^{m 2} \hat{D}_{p q r s} \eta_{r s k l m}^{m 2} d Y / Y, \quad \bar{P}_{i j}^{(1)}=\int_{Y} \eta_{p q i j}^{m 1}\left\{P_{p q}-\hat{D}_{p q r s} \eta_{r s}^{m 0}\right\} d Y / Y$, and $\bar{P}_{n i j}^{(2)}=\int_{Y} \eta_{p q i j n}^{m 2}\left\{P_{p q}-\hat{D}_{p q r s} \eta_{r s}^{m 0}\right\} d Y / Y$.

To estimate the macroscopic virtual work, the Hill-Mandel condition is usually employed in the second-order computational homogenization $[27,28]$. Under this condition, the virtual work of a macroscopic point is considered to be equal to the volume average of the virtual work of the RVE assigned to the macroscopic point. However, the virtual work of the RVE depends on the coordinates of the center of the RVE, $\Delta x_{i}^{M(I)}$, as shown in Eq. (B.2). In this study, the macroscopic virtual work is calculated by summing the virtual work of all the RVEs in the evaluation region:

$$
\frac{\delta W}{V}=\frac{1}{V} \sum_{I=1}^{N} \delta W^{m(I)}
$$

where $N$ is the number of RVEs in the evaluation region, which is estimated as

$$
N=\frac{V}{Y} \text {. }
$$

Here, we consider the first and second moments of the volume of a macroscopic evaluation region having a microscopically periodic structure as

$$
\begin{aligned}
& \int_{V} \Delta x_{i} d V=\sum_{I=1}^{N} \int_{Y}\left(\Delta x_{i}^{m 0(I)}+\Delta x_{i}^{m}\right) d Y=Y \sum_{I=1}^{N}\left(\Delta x_{i}^{M(I)}\right)+N \int_{Y} \Delta x_{i}^{m} d Y \\
& \int_{V} \Delta x_{i} \Delta x_{j} d V=\sum_{I=1}^{N} \int_{Y}\left(\Delta x_{i}^{M(I)}+\Delta x_{i}^{m}\right)\left(\Delta x_{j}^{M(I)}+\Delta x_{j}^{m}\right) d Y=Y \sum_{I=1}^{n}\left(\Delta x_{i 0}^{M(I)} \Delta x_{j}^{M(I)}\right)+N \int_{Y} \Delta x_{i}^{m} \Delta x_{j}^{m} d Y .
\end{aligned}
$$

Considering that the first moments on the macroscopic and microscopic scales are zero in Eq. (B.5), we obtain

$$
\sum_{I=1}^{N}\left(\Delta x_{i}^{M(I)}\right)=0
$$

Furthermore, the following relationship can be obtained from Eqs. (B.5), (B.6), and (B.7):

$$
\frac{Y}{V} \sum_{I=1}^{N}\left(\Delta x_{i}^{M(I)} \Delta x_{j}^{M(I)}\right)=J_{i j}-J_{i j}^{m},
$$

where

$$
\begin{aligned}
& J_{i j}=\frac{1}{V} \int_{V} \Delta x_{i} \Delta x_{j} d V, \\
& J_{i j}^{m}=\frac{1}{Y} \int_{Y} \Delta x_{i}^{m} \Delta x_{j}^{m} d Y,
\end{aligned}
$$

are the second-moments of the volume of the evaluation region and the RVE, respectively. Finally, the virtual work of the macroscopic evaluation region can be obtained as follows.

$$
\frac{\delta W}{V}=\left(\delta v_{i, j}^{0} \delta v_{i, j n}^{0}\right)\left(\left[\begin{array}{cc}
\bar{D}_{i j k l}^{(11)} & \bar{D}_{i j k l m}^{(12)} \\
\bar{D}_{n i j k l}^{(21)} & \bar{D}_{i j k l}^{(11)}\left(J_{n m}-J_{n m}^{m}\right)+\bar{D}_{n i j k l m}^{(22)}
\end{array}\right]\left\{\begin{array}{c}
v_{k, l}^{0} \\
v_{k, l m}^{0}
\end{array}\right\}-\left\{\begin{array}{c}
\bar{P}_{i j}^{(1)} \\
\bar{P}_{n i j}^{(2)}
\end{array}\right\}\right) .
$$


The macroscopic boundary problem can be solved using the macroscopic virtual work of the evaluation region.

\section{REFERENCE}

[1] Sutton, M. A., Walters, W. J., Peters, W. H., Ranson, W. F., McNeill, S. R., "Determination of displacements using an improved digital correlation method”, Image and Vision Computing, Vol. 1 (1983 ), pp.133-139.

[2] Sutton, M. A., Cheng, M., Peters, W. H., Chao, Y. J., McNeill, S. R., “Application of an optimized digital correlation method to planar deformation analysis”, Image and Vision Computing, Vol. 4 (1986), pp.143-150.

[3] Raabe, D., Sachtleber, M., Zhao, Z., Roters, F., Zaefferer, S., "Micromechanical and macromechanical effects in grain scale polycrystal plasticity experimentation and simulation” Acta Materialia, Vol.49 (2001), pp.34333441 .

[4] Parsons, E., Boyce, M. C., Parks, D. M., “An experimental investigation of the large-strain tensile behavior of neat and rubber-toughened polycarbonate”, Polymer, Vol.45 (2004), pp.2665-2684.

[5] Uchida, M. and Tada, N., "Sequential evaluation of continuous deformation field of semi-crystalline polymers during tensile deformation accompanied by neck propagation”, International Journal of Plasticity, Vol. 27 (2011), pp. 2085-2102.

[6] Uchida, M., Ueno, T., Abe, T. and Kaneko, Y., "Quantitative evaluation of the development of stress and strain fields using digital image correlation and finite element methods", Advanced Experimental Mechanics, Vol. 2 (2017), pp. 76-81.

[7] Uchida, M., Taniguchi, A. and Kaneko, Y., "Estimation of slip deformation of polycrystalline pure copper using digital image correlation method and crystalline plasticity theory”, Advanced Experimental Mechanics, Vol. 3 (2018), pp. 135-140

[8] Taniguchi, A., Maeyama, T., Uchida, M. and Kaneko, Y., "Macroscopic and microscopic non-uniform deformations of polycrystalline pure copper during uniaxial tensile test with high stress gradient”, Key Engineering Materials, Vol. 794 (2019), pp. 246-252.

[9] Avril, S., Pierron, F., Sutton, M. A. and Yan, J., "Identification of elasto-visco-plastic parameters and characterization of Lüders behavior using digital image correlation and the virtual fields method”, Mechanics of Materials, Vol. 40 (2008), pp.729-742.

[10] Wang, P., Pierron, F. and Thomsen, O. T., "Identification of material parameters of PVC foams using digital image correlation and the virtual fields method”, Experimental Mechanics, Vol. 53 (2013), pp.1001-1015.

[11] Cosserat, E and Cosserat, F, “Théorie des corps déformables”, Librairie Scientifique, A. Hermann et Fils, reprint 2009 by Hermann Librairie Scientifique, ISBN 978270566920 1, (1909), Paris.

[12] Toupin, R. A., "Elastic materials with couple-stress", Archive for Rational Mechanics and Analysis, Vol. 11 (1962), pp. 385-414.

[13] Mindlin, R. D., "Micro-structure in linear elasticity", Archive for Rational Mechanics and Analysis, Vol. 16 (1964), pp. 51-78.

[14] Mindlin, R. D. and Eshel, N. N., “On first strain-gradient theories in linear elasticity”, International Journal of Solids and Structures, Vol. 4 (1968), pp. 109-124.

[15] Aifantis, E. C., "On the role of gradients in the localization of deformation and fracture”, International Journal of Engineering Science, Vol. 30 (1992), pp.1279-1299.

[16] Aifantis, E. C., “Pattern formation in plasticity”, International Journal of Engineering Science, Vol. 33 (1995), pp.2161-2178. 
[17] Tomita Y., “Simulation of plastic instabilities in solid mechanics”, Applied Mechanics Reviews Vol. 47 (1994), pp. 171-205.

[18] Zervos, A., Papanastasiou, P. and Vardoulakis, I., "A finite element displacement formulation for gradient elastoplasticity”, International Journal for Numerical Methods in Engineering, Vol. 50 (2001), 1369-1388.

[19] Al-Rub, R. K. A. and Voyiadjis, G. Z., "A direct finite element implementation of the gradient-dependent theory”, International Journal for Numerical Methods in Engineering, Vol. 63 (2005), pp.603-629.

[20] Fleck, N. A. and Hutchinson, J. W., “A phenomenological theory for strain gradient effects in plasticity”, Journal of the Mechanics and Physics of Solids, Vol. 41 (1993), pp. 1825-1857.

[21] Fleck, N. A. and Hutchinson, J. W., "Strain Gradient Plasticity”, Advances in Applied Mechanics, Vol. 33 (1997), pp. 295-361.

[22] Gao, H. Huang, Y., Nix, W. D. and Hutchinson, J. W., "Mechanism-based strain gradient plasticity-I. Theory”, Journal of the Mechanics and Physics of Solids, Vol. 47 (1999), pp. 1239-1263.

[23] Gao, H. and Huang, Y., “Taylor-based nonlocal theory of plasticity”, International Journal of Solids and Structures, Vol. 38 (2001), pp. 2615-2637.

[24] Gurtin, M. E., “On the plasticity of single crystals: free energy, microforces, plastic-strain gradients”, Journal of the Mechanics and Physics of Solids, Vol. 48 (2000), pp.989-1036.

[25] Gurtin, M. E., "A gradient theory of single-crystal viscoplasticity that accounts for geometrically necessary dislocations”, Journal of the Mechanics and Physics of Solids, Vol. 50 (2002), pp.5-32.

[26] Gurtin, M. E., "On a framework for small-deformation viscoplasticity: free energy, microforces, strain gradients”, International Journal of Plasticity, Vol. 19 (2003), pp. 47-90.

[27] Kouznetsova, V., Geers, M. G. D. and Brekelmans, W. A. M., "Multi-scale constitutive modelling of heterogeneous materials with a gradient-enhanced computational homogenization scheme", International Journal for Numerical Methods in Engineering, Vol. 54 (2002), pp. 1235-1260.

[28] Kouznetsova, V., Geers, M. G. D. and Brekelmans, W. A. M., "Multi-scale second-order computational homogenization of multi-phase materials: a nested finite element solution strategy", Computer Methods in Applied Mechanics and Engineering, Vol. 193 (2004), pp. 5525-5550.

[29] Kaczmarczyk, Ł., Pearce, C. J. and Bićanić, N., "Scale transition and enforcement of RVE boundary conditions in second - order computational homogenization”, International Journal for Numerical Methods in Engineering, Vol. 74 (2008), pp. 509-522.

[30] Kaczmarczyk, Ł., Pearce, C. J. and Bićanić, N., "Studies of microstructural size effect and higher-order deformation in second-order computational homogenization”, Computers \& Structures, Vol. 88 (2010), pp. 1383-1390.

[31] Yuan, X., Tomita, Y. and Andou, T., "A micromechanical approach of nonlocal modeling for media with periodic microstructures”, Mechanics Research Communications, Vol. 35 (2008), pp.126-133.

[32] Bacigalupo, A. and Gambarotta, L., "Second-order computational homogenization of heterogeneous materials with periodic microstructure”, ZAMM - Journal of Applied Mathematics and Mechanics, Vol. 90 (2010), pp.796811.

[33] Bacigalupo, A., "Second-order homogenization of periodic materials based on asymptotic approximation of the strain energy: formulation and validity limits”, Meccanica, Vol. 49 (2014), pp.1407-1425.

[34] Lesičar T., Tonković, Z. and Sorić J., “A second-order two-scale homogenization procedure using $C^{1}$ macrolevel discretization”, Computational Mechanics, Vol. 54 (2014), pp. 425-441. 
[35] Uchida, M. and Tada, N., "Computational simulation of micro- to macroscopic deformation behavior of cavitated rubber blended amorphous polymer using second-order homogenization method”, Key Engineering Materials, Vol. 626 (2014), pp. 74-80.

[36] Uchida, M., Suzuki, K. and Kaneko, Y., "Computational simulation of scale-dependent non-uniform deformation behavior of polymer foam”, Key Engineering Materials, Vol. 725 (2016), pp. 456-461.

[37] Trovalusci, P., Ostoja-Starzewski, M. and Bellis M. L. D. and Murrali, A., "Scale-dependent homogenization of random composites as micropolar continua”, European Journal of Mechanics - A/Solids, Vol. 49 (2015), pp. 396-407.

[38] Coenen, E. W. C., Kouznetsova, V. G., Geers M. G. D., “Computational homogenization for heterogeneous thin sheets”, International Journal for Numerical Methods in Engineering, Vol. 83 (2010), pp. 1180-1205.

[39] Rosi, G. and Auffray A., “Anisotropic and dispersive wave propagation within strain-gradient framework”, Wave Motion, Vol. 63 (2016), pp. 120-134.

[40] Reda, H., Goda, I., Ganghoffer, J. F., L’Hostic, G. and Lakiss, H., “Dynamical analysis of homogenized second gradient anisotropic media for textile composite structures and analysis of size effects", Composite Structures, Vol. 161 (2017), pp. 540-551.

[41] Hu, R. and Oskay, C., "Spatial-temporal nonlocal homogenization model for transient anti-plane shear wave propagation in periodic viscoelastic composites", Computer Methods in Applied Mechanics and Engineering, Vol. 342 (2018), pp. 1-31.

[42] Li, J., "A micromechanics-based strain gradient damage model for fracture prediction of brittle materials - Part I: Homogenization methodology and constitutive relations”, International Journal of Solids and Structures, Vol. 48 (2011), pp. 3336-3345.

[43] Uchida, M. and Kaneko, Y., "Experimental modeling of nonuniform deformation in finite volume evaluation region of heterogeneous material”, Heliyon, Vol. 4 (2018), e00578, 21pp.

[44] Marty, J., Réthoré, J. and Combescure, A., “Experimental investigation of higher-order homogenization schemes under large strain”, International Journal of Solids and Structures, Vols. 88-89 (2016), pp. 263-273.

[45] Peirce, D., Asaro, R.J. and Needleman, A., "Material rate dependence and localized deformation in crystalline solids”, Acta Metallurgica, Vol. 31 (1983), pp.1951-1976.

[46] Hutchinson, J.W., "Bounds and self-consistent estimates for creep of polycrystalline materials”, Proceedings of the Royal Society of London A, Vol. 348 (1976), pp. 101-127. 